\title{
Effects of an added counter on keypecking maintained by a fixed-ratio schedule: Context effects
}

\author{
DAVID B. PEELE and C. B. FERSTER \\ The American University, Washington, D.C.
}

\begin{abstract}
Under fixed-ratio reinforcement, an added counter weakened performance to a greater extent when the added counter alternated with an identical fixed-ratio schedule (without the counter) in a multiple schedule. The results were interpreted as indicating an increase in the discriminative control by the added counter, which was caused by the contrast with the interpolated ratio requirement without the counter. The results confirm and extend earlier reports that fixed-ratio performance is substantially weakened by amplification of the discriminative control of performance by response count.
\end{abstract}

A previous experiment gave evidence that the postreinforcement pause on a fixed-ratio schedule was longer with an added counter-a slit on the response key that provided an exteroceptive stimulus that was small at the start of the fixed ratio and large at completion of the ratio requirement (Ferster \& Peele, 1980). In that experiment, the effect of the added counter was studied in a multiple schedule in which the added stimulus grew with response count in one component of the multiple schedule, and remained fixed (either at a small or a large opening size) during the alternating component of the multiple schedule. The added counter increased the pause after reinforcement, relative to the performance without the counter. The extent of the attenuation of fixed-ratio performance depended, however, on whether the added stimulus was fixed at the small or the large position during the component without the counter. This was necessitated because the components were programmed on the same key. The added counter weakened performance to a greater degree when the alternating schedule, without it, was occasioned by the large slit size. This finding was interpreted as indicating induction between the two components of the multiple schedule. The reinforcement of pecks at the large slit size, without the added counter, provided a contrast of conditions at the beginning of the fixed ratio that accented its unoptimal control of behavior. The reinforcement at the small stationary slit reduced the unoptimality of the small size of the added counter at the start of the fixed ratio and, hence, lessened the pausing emitted with the added counter.

In the present experiment, we examined the effect of the added counter by using two keys to eliminate these

The research described in this article was conducted prior to C. B. Ferster's death in February 1981, and was supported in part by National Science Foundation Grant BNS-8309045 to The American University. Correspondence should be addressed to David B. Peele, NSIEnvironmental Sciences, P.O. Box 12313, Research Triangle Park, NC 27709. complex interactions and to more accurately measure the attenuation and the conditions that govern it.

\section{METHOD}

\section{Subjects}

Two naive adult male Silver King pigeons, maintained at $80 \%$ of their free-feeding weights, served as subjects. The total daily ration of food was delivered during experimental sessions. Experimental sessions were conducted until 60 reinforcements occurred; the food-magazine duration was adjusted between 4 and 5 sec to maintain a constant weight.

\begin{abstract}
Apparatus
The experiment was carried out in a two-key, standard pigeon chamber, as described by Ferster (1953). The added counter was a slit, $3.2 \mathrm{~mm}$ wide and back-illuminated, that grew from $3.2 \mathrm{~mm}$ long at the start to $28.6 \mathrm{~mm}$ at the completion of the fixed-ratio requirement. The two keys were centered at equal distances from the food hopper. The added counter key was illuminated with white light, and the key without the counter was illuminated with yellow light. The added counter was driven by an automatic electric stepping relay that operated through gear reduction. The stepper drove a wedge-shaped mask across the key, increasing the height of the slit with each response. The rate of growth was linear. A counterweight returned the slit to the beginning position when an electromagnetic clutch was actuated. Reset time was less than $0.5 \mathrm{sec}$. The delivery of food was linked mechanically to a switch on the added counter, to ensure that the added stimulus was always at the maximum position at the moment of reinforcement. The data were recorded on a cumulative recorder and a Sodeco counter, which printed out individual interreinforcement times.
\end{abstract}

\section{Procedure}

Keypecks were shaped by successive approximations to the desired response, and then reinforced continuously on alternate keys with the added counter held at the maximum position. Contingencies were indicated by illuminating the respective keys. The fixed-ratio requirement was then increased to the terminal value of 200 in 10 steps over a period of five sessions. During this time, components alternated between keys, each with the same ratio requirement. During training, the added counter remained stationary at the large slit size. Thereafter, three stages followed each other in continuous, simple alternation, each in effect for a full session. Stage 1 was a multiple schedule in which a fixed-ratio 200 (FR200) alternated between the added counter key and the key without the counter. Stage 1 was held in effect for a total of 19 sessions. In the second and third stage, only the key without the added 
counter or the key with the added counter, respectively, was operative while the alternate key was dark. Stages 2 and 3 were held in effect for nine sessions each. Since the fixed-ratio performance in components with and without the added counter was uniformly a pause after reinforcement followed by an abrupt shift to the prevailing fixed-ratio running rate, the interreinforcement time was considered the major datum. The effects of treatment on performance were assessed by determining mean interreinforcement times (in seconds) for performances with and without the added counter.

\section{RESULTS AND DISCUSSION}

The results for both subjects are shown in Figure 1, which illustrates the interreinforcement time with and without the added counter when the schedules were components of the multiple schedule and when the schedules were programmed alone as a simple schedule. The left panel shows the fixed-ratio performance with the added counter when it was programmed as part of a multiple schedule (open bars) and when it was programmed as a simple schedule (filled bars). The right panel shows analogous fixed-ratio performance on the key without the added counter. In all cases, the interreinforcement time was substantially longer with the added counter. Overall, mean interreinforcement times with, as opposed to without, the
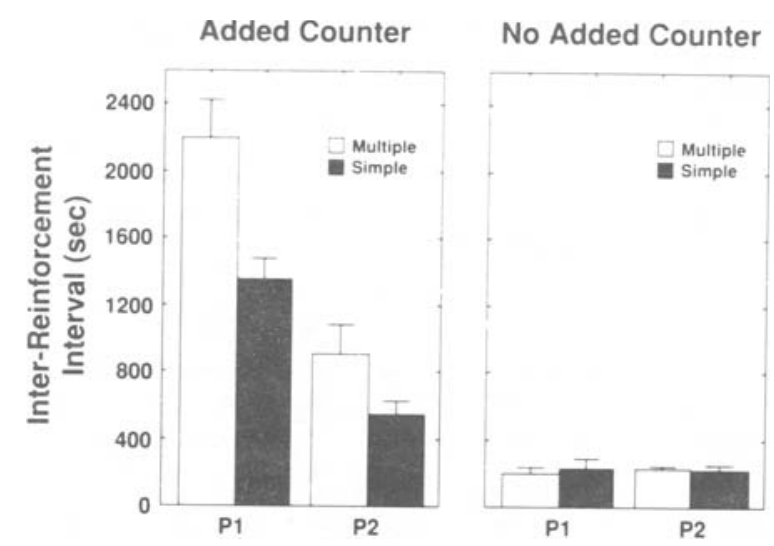

Figure 1. Mean interreinforcement intervals obtained on a fixedratio 200 schedule of reinforcement, with and without an added counter. Values for performance with the added counter, shown in the left panel, are illustrated for instances where contingencies were arranged as either a simple (filled bars) or a multiple (open bars) schedule. A similar data set is shown in the right panel for performance maintained by an identical fixed-ratio schedule without the added counter. Each bar represents group mean $( \pm S E)$ performance for each of 2 pigeons. added counter were 1,250 versus $226 \mathrm{sec}$, respectively, or a 5-fold increase produced by the addition of the added counter. Performance maintained by FR200 without the added counter (right panel) was roughly identical in both multiple and simple schedule conditions. This was not the case for performance on the FR200 with the added counter. As shown in the left panel of the figure, both birds took substantially longer to complete the ratio in the context of the multiple-schedule presentation. The increase in interreinforcement time resulting from contextual effects for Pigeons 1 and 2 represents increases of $62 \%$ and $65 \%$, respectively.

The results confirm and extend the findings reported by Ferster and Peele (1980) that the added counter weakens performance maintained by a fixed-ratio schedule. In the present study, we avoided the confounding effects of induction reported in the earlier experiment by recording performance with and without the added counter on two separate response keys. The novel finding in the present experiment was that fixed-ratio strain accompanying performance with the addition of an added counter was enhanced by alternating a fixed-ratio component with the added counter with a similar fixed-ratio requirement without the added counter. By virtue of alternation with an identical fixed-ratio schedule in the absence of explicit feedback of count, the added counter acquired enhanced discriminative control over responding. One interpretation of the data is that the added counter eliminates the ambiguity that is normally present in the bird's own behavior when that behavior is the controlling discriminative stimulus. In this manner, the performance maintained by the fixed ratio without the added counter, in relation to that maintained by the fixed ratio with the added counter, was functionally similar to the performance maintained by a variable-ratio schedule (Ferster \& Skinner, 1957).

\section{REFERENCES}

Ferster, C. B. (1953). The use of the free operant in the analysis of behavior. Psychological Bulletin, 50, 263-274.

Ferster, C. B., Peele, D. B. (1980). The contribution of an added counter to a fixed-ratio schedule. Journal of the Experimental Analysis of Behavior, 34, 93-105.

FERSTER, C. B., \& SKINNER, B. F. (1957). Schedules of reinforcement. New York: Appleton-Century-Crofts.

(Manuscript received September 14, 1989.) 